\title{
Research On The Buttress-Warehouse Retaining Wall Based On Centrifugal Model Test
}

\author{
Ying $\mathrm{Nie}^{1,2, \mathrm{a}}$,Xuepeng Ling ${ }^{3, \mathrm{~b}}$, Fenghan $\mathrm{Hu}^{1,2, \mathrm{c}}$, \\ Qingsong Zhang ${ }^{3, d}$,Xiaofeng Zhao ${ }^{1,2, e}$ and Bo Liang ${ }^{3, f}$ \\ 1. CISDI Research \& Development Co. Ltd., Chongqing 401133, P.R. China \\ 2. CISDI Architectural Design \& Research Institute, Chongqing 401133, P.R. China \\ 3. School of Civil Engineering, Chongqing Jiaotong University, Chongqing 400074, P.R. China \\ aying nie@cisdi.com.cn, b1140319141@qq.com, ${ }^{\mathrm{c} f e n g h a n . h u @ c i s d i . c o m . c n, ~}$ \\ d752750879@qq.com, exiaofeng.zhao@cisdi.com.cn, 'liang_laoshi@126.com
}

Keywords: the buttress retaining wall; soil pressure; deformation characteristic; geotechnical centrifuge model experiment

\begin{abstract}
The buttress-warehouse retaining wall is the combination of buttress retaining wall and warehouse retaining wall. In this paper, with different widths of buttress, the wall's soil pressure and deformation characteristics have been discussed in geotechnical centrifuge model experiment. The results show that 1 . the vertical plate's soil pressure is less than the Coulomb active soil pressure; 2. the vertical deformation of the filling (settlement) is greater than the horizontal;3. the wall is inclined outward rotation around the buttress plane.
\end{abstract}

\section{Introduction}

Geotechnical centrifuge model experiment - the reduction of the size of prototype to $1 / \mathrm{n}$ and the addition of the acceleration of gravity increases to $\mathrm{ng}$ - is designed to simulate a prototype structure of the stress and deformation in the actual process of construction and operation. It can make sure that both the model and the prototype are working in the environment, which they own the same mechanical properties .

Zurun Yue ${ }^{[1]}$ has discussed the relationship between the cohesive soil of soil pressure distribution and the displacement of the wall by geotechnical centrifuge model experiment. Hong Liu ${ }^{[2]}$, based on the similarity theory of model experiment, conducted a centrifugal model experiment for the 104m high filling foundation of Jiuzhaigou airport, and explored the settlement of the soil during construction and operation. Hao $\mathrm{Li}^{[3]}$ made a centrifugal experiment model to simulate the weight of the shoulder wall, and studied the effect of the deformation of the soil with different displacement modes. It can be seen that the centrifugal model experiment technology has gained considerable experience in the research field of high retaining wall.

The new type of retaining structure, which is represented by buttress retaining wall, has developed rapidly in the past 20 years. Because of its simple construction, convenient construction, small area and light weight, the buttress retaining wall is widely used in engineering. The research of buttress retaining wall is still hot in China. Yuanjian Mai ${ }^{[4]}$ based the monitoring results of buttress retaining wall in Guangzhou Huangpu new port, found that buttress significantly reduces vertical plate earth pressure, and revealed the distribution of the earth pressure distribution is nonlinear. Yuyou Wei ${ }^{[5]}$ 
adopted model experiments to verify the soil pressure of buttress retaining wall in Guangzhou Huangpu new port, and found that the earth pressure was smaller than Rankine earth pressure. Duoyin Wang ${ }^{[6]}$ studied the soil pressure distribution of buttress retaining wall by three-dimensional finite element software.

The technique of retaining wall retaining wall is relatively mature in China. However, when the height of filling is over $10 \mathrm{~m}$, the deformation and the stability of the wall can be easily controlled.

The combination of the buttress retaining wall and warehouse format retaining wall is aim to solve the problem of the deformation the stability of the wall under the high filling environment

In this paper, the combination of the buttress retaining wall and warehouse format retaining wall which the height is $30 \mathrm{~m}$ is studied the pressure and the deformation characteristics of the wall by Geotechnical centrifuge model experiment.

\section{Geotechnical Centrifuge Model Experiment}

Experiment Equipment. The tlj-60 geotechnical centrifuge in the geotechnical centrifuge laboratory of Chongqing jiaotong university was used in this experiment. The effective radius of the equipment is 2 meters; Model box size: $70 \mathrm{~cm} \times 40 \mathrm{~cm} \times 60 \mathrm{~cm}$.

The Design of Model Size. This experiment mainly simulates the wall which the height is $30 \mathrm{~m}$. The filling after the wall is the horizontal position and up to the top of wall. The dimensions of each component of the wall are shown in the Table 1 .

Table 1 The dimensions of model

\begin{tabular}{ccccc}
\hline $\begin{array}{c}\text { The height of the } \\
\text { wall }(\mathrm{cm})\end{array}$ & $\begin{array}{c}\text { Model } \\
\text { ratio }\end{array}$ & $\begin{array}{c}\text { The length of } \\
\text { warehouse }(\mathrm{cm})\end{array}$ & $\begin{array}{c}\text { The width of } \\
\text { buttress }(\mathrm{cm})\end{array}$ & $\begin{array}{c}\text { The length of the heel } \\
\text { plate }(\mathrm{cm})\end{array}$ \\
\hline 33 & $1: 90$ & 6 & 9 & 12 \\
\hline
\end{tabular}

The Simulation of The Model Material. The model is required material which is similar to the material density of the prototype. And the model 'material own a high stiffness and strength. The density of aluminum and concrete is about $2.0 \sim 2.8 \times 10^{3} \mathrm{~kg} / \mathrm{m}^{3}$

$$
\frac{E_{m 1} \delta_{m 1}^{3}}{12\left(1-v^{2}{ }_{m 1}\right)}=\frac{E_{m 2} \delta^{3}{ }_{m 2}}{12\left(1-v_{m 2}^{2}\right)}
$$

In the Eq. $1, E_{m 1}$ is the modulus of elasticity of a prototype, $E_{m 2}$ is the modulus of elasticity of aluminum, $\delta_{m 1}$ is the thickness of a prototype with the model ratio of $1 / 90, \delta_{m 2}$ is the thickness of aluminum, $v_{m l}$ is the poisson ratio of a prototype. $v_{m 2}$ is the poisson ratio of aluminum.

Table 2 The material parameter of model

\begin{tabular}{ccc}
\hline material & the modulus of elasticity $(\mathrm{GPa})$ & poisson ratio \\
\hline reinforced concrete & 34 & 0.167 \\
\hline aluminum & 70 & 0.34 \\
\hline
\end{tabular}

The thickness of a prototype is designed about $900 \mathrm{~mm}$ and the model ratio is $1 / 90$. With the calculation, $\delta_{m l}$ is $10 \mathrm{~mm}$. So, with the formula 1, the $\delta_{m 2}$ can be calculated. Finally, the $\delta_{m 2}$ is $8 \mathrm{~mm}$. The connection of the components is maintained by hexagon bolt. The picture of the model which the width of buttress is $9 \mathrm{~cm}$ is shown in the Fig. 1 


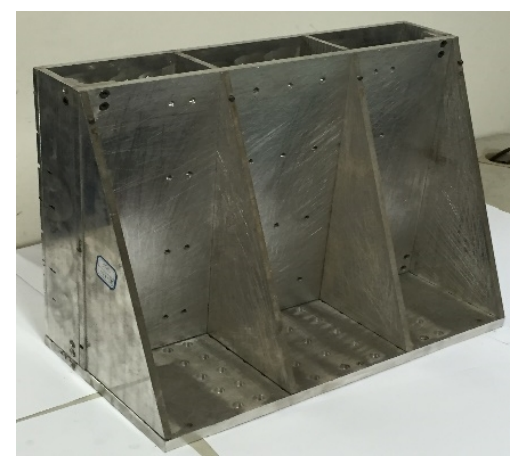

Fig. 1 the model of buttress-warehouse retaining wall

The Simulation of The Filling Material. The gravelly soil is chosen as the model filling and collected in Tushan car depot of Chongqing light rail transit line. Optimum moisture content and maximum dry density of the filling, obtained by compaction test, are respectively $10.8 \%$ and $2.04 \mathrm{~g} / \mathrm{cm}^{-3}$. The integrated internal friction angle of the filling is about $30^{\circ}$ in the shear test.

Calculation Method of Earth Pressure Theory. According to Highway subgrade design specification of Chinese, it is advised that the theory of imagination wall back can be applied to calculate the earth pressure of the buttress retaining wall. The theory ,described as in the picture, is that lining the top of the wall (point A) and the heel of the wall (point B) is selected as the imagination wall back of the buttress retaining wall, then Coulomb active earth pressure theory can be applied in the wall back .

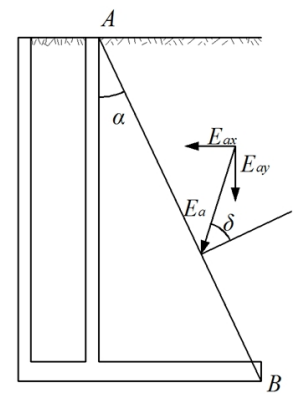

Fig. 2 The calculation model

The soil surface is horizontal which can be found in the Fig. 2.

$p_{a}=K_{a} \cdot \gamma \cdot z$

$K_{a}=\frac{\cos ^{2}(\varphi-\alpha)}{\cos ^{2} \alpha \cos (\alpha+\delta)\left[1+\sqrt{\frac{\sin (\varphi+\delta) \sin (\varphi-\beta)}{\cos (\alpha+\delta) \cos (\alpha-\beta)}}\right]^{2}}$

$E_{a x}=E_{a} \cos (\alpha+\delta)$

In the Eq. 2 Eq. 4, $E_{a}$ represents the active earth pressure of imagination wall back, $E_{a x}$ represents the horizontal active earth pressure of imagination wall back, $K_{a}$ represents coefficient of active earth pressure, $\gamma$ represents the filling weight which is about $19.2 \mathrm{kN} / \mathrm{m}^{3}$ in this experiment, $\psi$ represents integrated internal friction angle of the filling, $\delta$ represents integrated internal friction angle between filling and the imagination wall back, $\alpha$ represents the angle between vertical plate and the imagination wall back, $\beta$ represents the angle between filling surface and the horizontal plane of the top of the wall. Test equipment in laboratory. soil pressure cells ,produced by the Chinese physics institute, are adopt to measure the soil pressure of vertical plate. The parameter of the soil pressure cells is shown in the Table3. 
Table 3 The parameter of soil pressure cells

\begin{tabular}{cccccc}
\hline type & Range(kPa) & Power supply & full-span output voltage & Diameter(mm) & Thickness to diameter ratio \\
\hline I & 2000 & $6 \sim 12 \mathrm{~V}$ & $2000 \mathrm{mV}$ & 14 & 0.14 \\
\hline II & 500 & $6 \sim 12 \mathrm{~V}$ & $2000 \mathrm{mV}$ & 8 & 0.25 \\
\hline
\end{tabular}

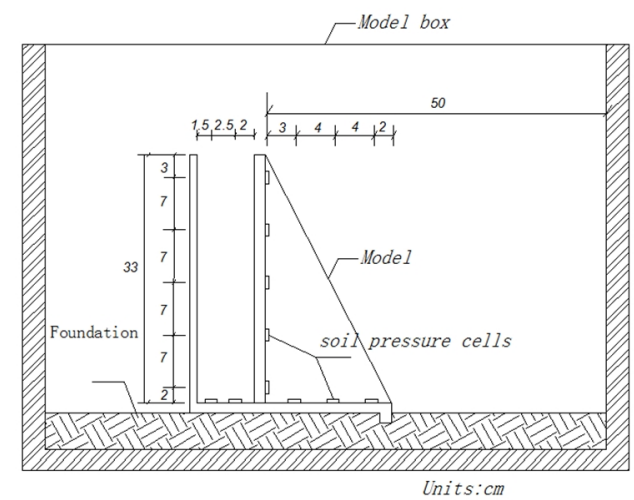

Fig. 3 The arrangements of soil pressure cells

The soil pressure of vertical plate the horizontal plane should be measured in this experiment. According to the corresponding references of soil pressure cell, the spacing of the cells should be over 1.5 times the cell diameter. And, with the consideration of the model wall's actual size, the arrangement of soil pressure cells is shown in the Fig. 3.

\section{The Filling Process.}

Construction of Embankment. The foundation soil is designed to divide into two layers. The height of the top is $2 \mathrm{~cm}$, and the bottom, galling the surface, is $3 \mathrm{~cm}$. The compaction of the foundation is controlled about $95 \%$. To make sure the wall model can be set on the embankment horizontal, tenon must be pre-buried in the top layer of the embankment.

Construction of Embankment. After the model wall is successfully set on the foundation, the gravelly soil is filled with 5 layers. The thickness of layer is about $6 \mathrm{~cm}$ and the layer surface should be galled. The compaction of the filling is controlled about $85 \%$.

Filling Displacement Monitoring. In order to reflect the displacement of the filling visually, the side view of the filling is meshed generation which size is $4 \mathrm{~cm} \times 4 \mathrm{~cm}$. The grid points are marked with a small piece of paper.

\section{Discussion}

The Calculation of Soil Pressure. the soil pressure cells of the vertical plate can be made in the Table4 shown in the Fig. 4.

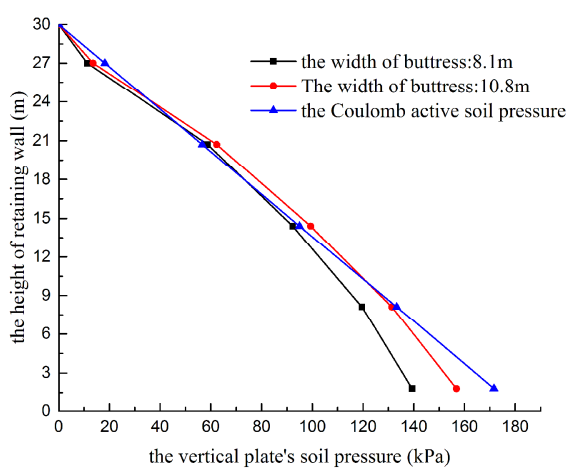

Fig. 4 The distribution of soil pressure 
Owing to the friction between buttresses and the filling, the rate of increase vertical plate's pressure reduces as the decreasing height of the wall. And its minimum appears near the heel of the wall. The unit width of the active soil pressure value is the corresponding area of distribution of earth pressure.

Variation of earth pressure $\beta$ (Eq. 5)is defined as ratio of the difference between the vertical plate's pressure $F_{x}$ and the Coulomb active soil pressure $F_{a}$ to the Coulomb active soil pressure $F_{a}$.

$\beta=\frac{F_{x}-F_{a}}{F_{a}} \times 100 \%$

Table 4 The soil pressure

\begin{tabular}{cccc}
\hline \multirow{2}{*}{ The wall height of $30 \mathrm{~m}$} & \multicolumn{2}{c}{ The width of buttress $(\mathrm{m})$} & \multirow{2}{*}{ Coulomb active soil pressure value } \\
\cline { 2 - 3 } & $8.1 \mathrm{~m}$ & $10.8 \mathrm{~m}$ & \\
\hline Soil pressure $(\mathrm{kN} / \mathrm{m})$ & 2368.7 & 2583.0 & 2738.9 \\
\hline$\beta$ & $-13.52 \%$ & $-5.25 \%$ & -
\end{tabular}

As the Table4 show, the vertical plate's pressure $F_{x}$ is less than the Coulomb active soil pressure $F_{a}$. The reason why is that the calculation of Coulomb active soil pressure fails to take consideration of the friction between buttresses and the filling.

Filling Deformation Analysis. The deformation of the filling can be made into the Fig.5 Fig. 8

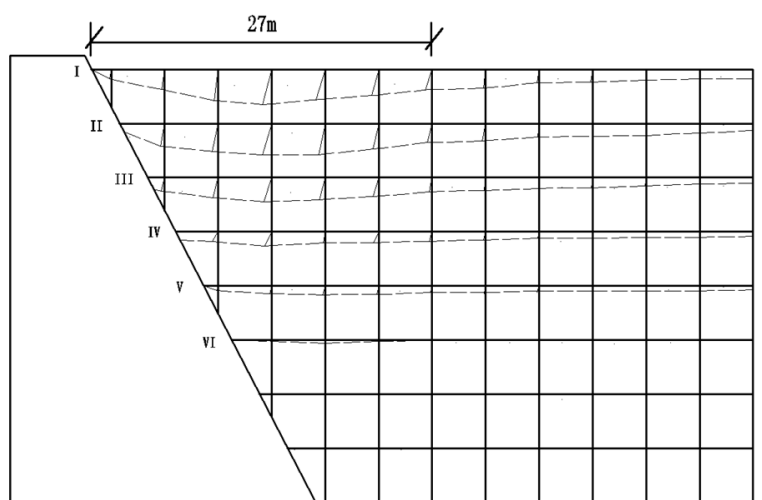

Fig. 5 The deformation of the filling $(8.1 \mathrm{~m})$

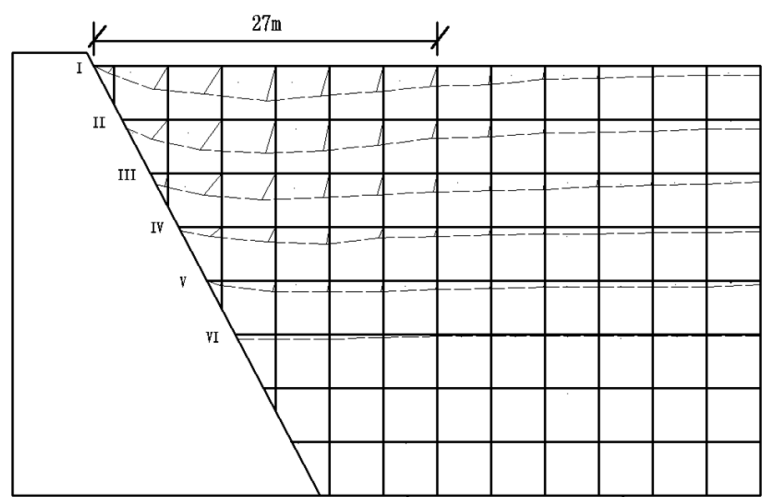

Fig. 6 The deformation of the filling(10.8m)

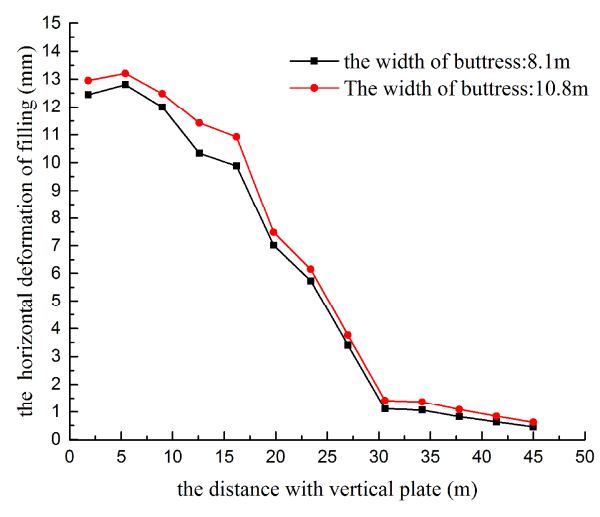

Fig. 7 The horizontal deformation of range I

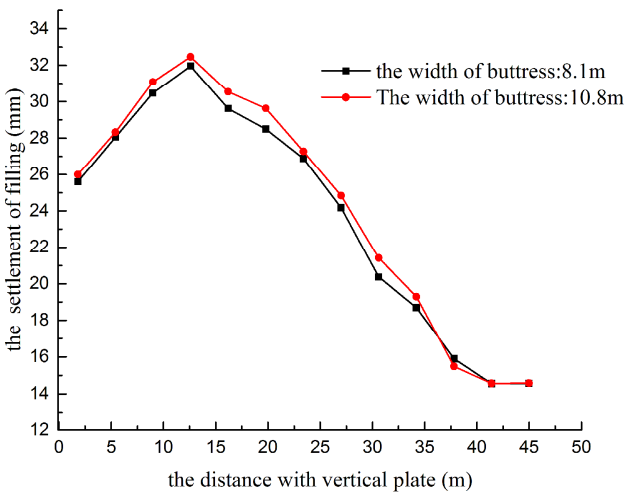

Fig. 8 The settlement of of range I

Seeing Fig.5 Fig.8, we get the conclusions as follow.

1.It is evident that the vertical of deformation (settlement) is greater than the horizontal.

2.The wall is inclined outward rotation around the buttress plane. Because the deformation of the top wall is over than the bottom. The deformation of I VI region, which correspond the height of wall 
ranging from $11 \mathrm{~m}$ to $30 \mathrm{~m}$, is outstanding. Because of the construction by layers, the compaction of low layer of filling is always greater than the upside. Finally, with the reduction of the height of the wall, the deformation decreases.

3.The horizontal deformation of filling reduces from the left side to right side and the settlement trend is showing a "V" type in the same height of the wall. Take the range I deformation for example, the settlement is nearly symmetrical within the range of $0 \sim 27 \mathrm{~m}$ from the vertical plate, while there is a downward trend within the range of $27 \sim 45 \mathrm{~m}$ from the vertical plate.

There are two reason leading to the settlement. One is the self -weight of filling. Another is that the wall rotate $\mathrm{s}$ around the inclined plane of the buttresses, which ,to a certain extent, promotes the settlement of the filler after the wall. The inclined buttresses has an upward effect on a small portion of the filling which near the buttresses, which leads to these filling's settlements reduce. And this phenomenon little affect the filling within the range of $27 \sim 45 \mathrm{~m}$ from the vertical plate. Finally, the settlement trend is showing a "V" type in the same height of the wall.

\section{Conclusions}

In this paper, with different widths of buttress, the study of the buttress-warehouse retaining which height is $30 \mathrm{~m}$ is carried out by the centrifugal model experiment.

1. The vertical plate's pressure is less than the Coulomb active soil pressure. The reason why is that the friction between buttresses and the filling leads to reduce the pressure.

2. The vertical deformation of the filling (settlement) is greater than the horizontal.

3. The wall is inclined outward rotation around the buttress plane.

\section{Acknowledgments}

The authors are grateful to the Science Program of Chongqing Municipal Commission of UrbanRural Development (No: 2016-0001) and the Foundation of CISDI Research \& Development Co. Ltd.(90270341-KJ-002) for the financial support for this research project.

\section{References}

[1] Zurun Yue, Shide Zhang. Study On Retaining Wall Pressure of Compacted Clay Filling In The Centrifugal Model Experiment. Yantu Gongcheng Xuebao, 1992, 14(6): 89-95(In Chinese).

[2] Hong Liu, Zhuo-Yuan Zhang, Wen-Xi Han. Centrifugal Model Technique of High Embankment. Geological Science And Technology Information, 2005, 24(1): 103-106(In Chinese).

[3] Hao Li, Qiang Luo, Liang Zhang, et al. Centrifugal model tests on shoulder balance weight retaining wall with various motion modes. Chinese Journal of Geotechnical Engineering, 2015, (4): 675-682(In Chinese).

[4] Yuanjian Mai. On The Calculating Of Earth Pressure Acting On Counterfort Structure.Chinese Journal of Geotechnical Engineering, 1982, 4(1): 24-33(In Chinese).

[5] Yuanyou Wei. The Measurement And Calculation Of Soil Pressure of Buttress Retaining Wall. Yantu Gongcheng Xuebao, 1987, 9(6): 89-100(In Chinese).

[6] Duo-Yin Wang, You-Ren Wu, Shi-Liang Zhou. Analysis of Behaviors of Earth Pressure Behind Counterfort Retaining Wall with Finite Element Method. China Harbour Engineering, 2006, (2): 14-17(In Chinese). 